\title{
Choroidal vasculitis in acute posterior multifocal placoid pigment epitheliopathy
}

\author{
Richard F Spaide, Lawrence A Yannuzzi, Jason Slakter
}

\begin{abstract}
A 24-year-old Caucasian female presented with acute posterior multifocal placoid pigment epitheliopathy (APMPPE) and associated infiltration round some of the larger choroidal blood vessels. This infiltration dissipated as the patient's clinical condition improved and did not induce any permanent alteration of the overlying retinal pigment epithelium. We suggest that the infiltration round the choroidal vessels was due to a choroidal vasculitis. The finding of choroidal inflammation in this case lends support to the hypothesis that choroidal vasculitis is an underlying pathological process in APMPPE.
\end{abstract}

In $1968 \mathrm{Gass}^{1}$ described three young women who developed a rapid, but transient, loss of visual acuity associated with multiple yellow-white placoid lesions at the level of the retinal pigment epithelium (RPE). Although the presence of choroidal involvement could not be ruled out, the disorder was presumed to involve primarily the RPE and was named with the descriptive term, acute posterior multifocal placoid pigment epitheliopathy (APMPPE).' The concept that a choroidal vasculopathy played a part in the pathogenesis of APMPPE was the first made by Van Buskirk and associates. ${ }^{2}$ Deutman and associates ${ }^{3}$ suggested that an acute inflammation of the choriocapillaris might be the initial incident in APMPPE and the RPE changes were a subsequent manifestation. Several systemic findings associated with APMPPE involve a vasculitis retinal vasculitis, ${ }^{+7}$ cerebral vasculitis, ${ }^{89}$ and erythema nodosum ${ }^{236}$ - which seems to support this hypothesis.

Direct evidence of choroidal vascular inflam-

LuEsther Mertz Retina Research Fund and the Macula Foundation of the Manhattan Eye, Ear, and Throat Hospital, New York

R F Spaide

L A Yannuzzi

J Slakter

Correspondence to:

Richard F Spaide, MD, Retin Service, Manhattan Eye, Ear, and Throat Hospital, $210 \mathrm{E}$ 64th Street, New York, NY 10021 , USA.

Accepted for publication 26 October 1990

Figure 1 A mation has remained elusive in APMPPE because the placoid lesions, as well as the melanin in the RPE and the melanocytes of the choroid, usually block visualisation of the choroidal vessels. We recently examined a young woman with APMPPE who had clinically discernible infiltrates of some of her peripheral choroidal vessels.

\section{Case report}

A previously healthy 24-year-old fair-skinned, red-haired woman was referred for ophthalmological examination because of a four-day history of decreased vision in the right eye associated with severe headaches. She had a visual acuity of 20/60 in the right eye and 20/20 in the left. Fundus examination revealed multiple yellow-white placoid lesions at the level of the retinal pigment epithelium in both eyes (Figs 1A, B). The lesions were hypofluorescent in the early stages of the fluorescein angiogram but showed late staining. Two weeks after presentation the headaches stopped, but her visual acuity declined to counting fingers at 4 feet $(120 \mathrm{~cm})$ in the right eye and $20 / 400$ in the left, secondary to bilateral foveal lesions. Fluorescein angiography at this time showed larger choroidal vessels in the subacute lesions (Fig 2), very slow filling of the choriocapillaris, and late staining of the lesions. There were no placoid lesions anterior to the mid-periphery.

In the periphery of both eyes some of the choroidal vessels were surrounded with patchy, segmental yellowish infiltration (Figs $3 \mathrm{~A}, \mathrm{~B}$ and 4A, B). Several of the more prominently involved vessels clearly led to the ampulla of the vortex veins; in other involved choroidal vessels the classification of artery or vein could not be made.

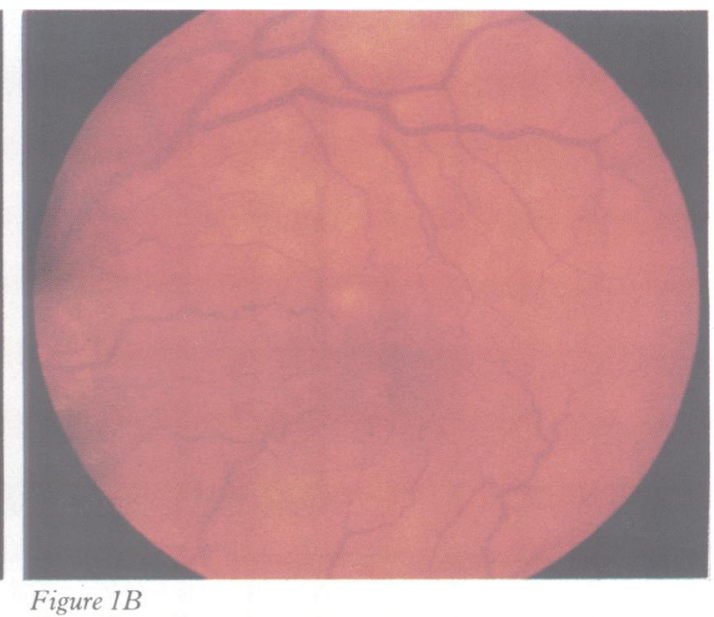
Figure IB

Figure 1 Fundus photographs of the patient with APMPPE on the day of presentation. A: Right eye. B: Left eye. 


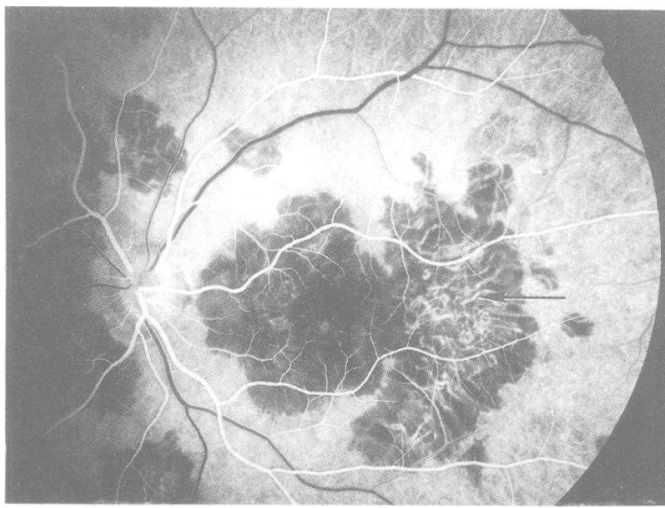

Figure 2 Early phase fluorescein angiogram of subacute lesions in the same patient two weeks after presentation. The subacute lesions were characterised by resolution of the dense placoid lesions, atrophy of the involved retinal pigment epithelium, and unveiling of the choroidal circulation. In such lesions the larger vessels could be seen during the early stages of the fluorescein angiogram, but the blush due to the filling of the choriocapillaris was not present.

The choroidal infiltration did not appear to involve the RPE by slit-lamp biomicroscopic examination with a three-mirror contact lens.

Over the next seven weeks both the placoid lesions and the peripheral choroidal vascular changes slowly resolved in parallel. Ten weeks after presentation the visual acuity was $20 / 40$ in both eyes. The choroidal infiltrates had resolved in both eyes without detectable damage to the larger choroidal vessels or any discernible change of the overlying RPE.

During the course of her illness she was not noted to have any abnormalities of the external eye, anterior chamber, vitreous, or retinal vessels. Laboratory tests included a complete blood count with differential count, liver function tests, thyroid function tests, electrolytes, Lyme disease titres, and a chest roentgenogram. With the exception of a borderline low serum iron level, all tests gave normal results.

\section{Discussion}

Infiltration round retinal vessels commonly occurs in conditions that cause a retinal vasculitis.
We suggest that the infiltration round the choroidal vessels in our case of APMPPE represents a choroidal vasculitis or an accumulation of the by-products of inflammation round the involved choroidal vessels. A total of six patients are mentioned in four previous reports concerning retinal vasculitis associated with APMPPE, ${ }^{+7}$ and in each instance the involved vessels were veins. In our patient several of the involved choroidal vessels could be identified as veins, but no identification could be made about other vessels.

While choroidal inflammatory disease has been implicated in the pathogenesis of APMPPE, clinical signs of choroidal vasculitis in the acute stages of the disease have not been described. Several factors probably contributed to our visualisation of the choroidal infiltrates in the periphery, but not in the posterior pole. In the mid and far peripheral fundus the RPE is more attenuated and the larger choroidal vessels lie closer to the RPE than in the posterior pole. ${ }^{10}$ The clinical focus of the disease, as implied by the name, is in the posterior pole, where the placoid lesions obscure the underlying choroidal vessels.

Many reported cases of APMPPE have had the prodromal symptoms of headache, fever, malaise, and myalgia. ${ }^{11-19}$ These symptoms were ascribed by the patients or the investigators to a cold, influenza, or other viral infection. It has been presumed that these prodromal infections, directly or indirectly, caused an inflammation of the choroidal vessels, with APMPPE as a consequence. Although antecedent viral infections frequently have been implicated as the cause of APMPPE, the evidence for this supposition is far from compelling. In only one case of APMPPE was a virus, adenovirus type 5 , isolated, ${ }^{20}$ but this patient developed evidence of the viral infection 19 days after being affected by APMPPE, raising the suspicion that this association may have been coincidental. Another case of APMPPE associated with acute nephritis had a positive immunofixation test for the virus of epidemic nephritis, ${ }^{21}$ but there was no increase in the antibody titre during the course of the disease.

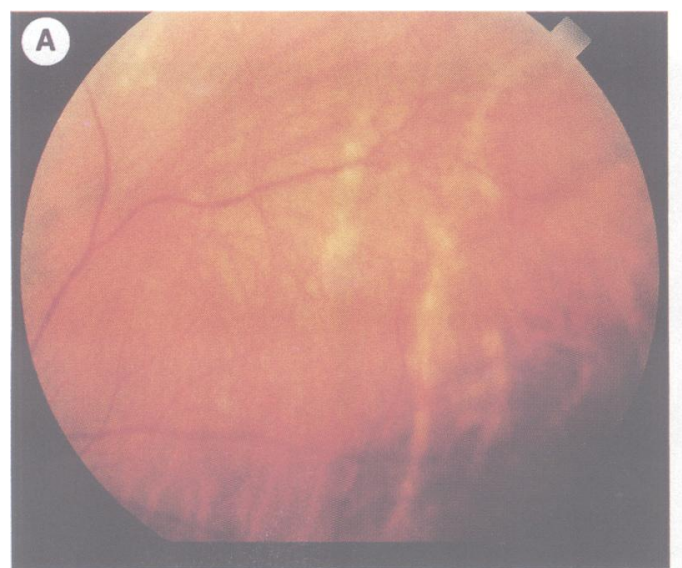

Figure $3 A$

Figure 3 Choroidal inflammation in APMPPE. A. Infiltration round a choroidal vessel as seen three weeks after initial presentation. B: Disappearance of the choroidal inflammation 10 weeks after presentation, with no residual damage to the retinal pigment epithélium.

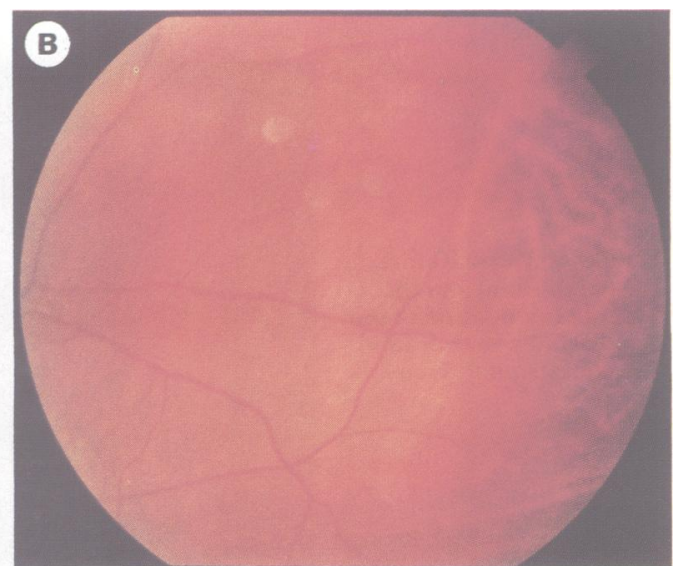

Figure $3 B$ 


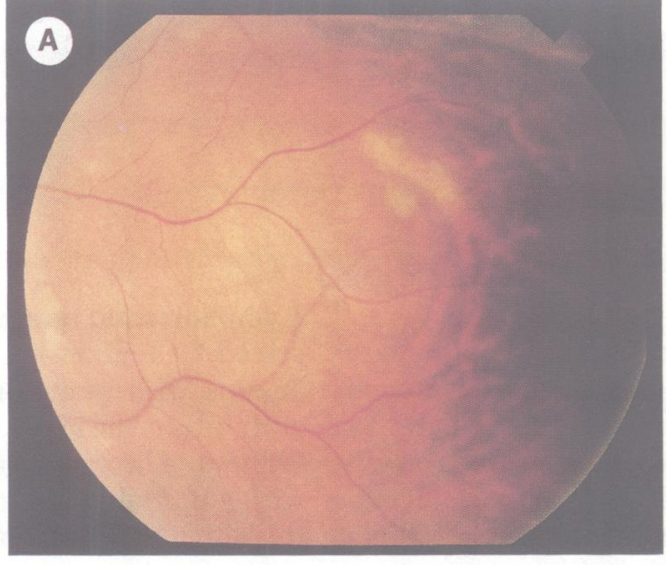

Figure 4 A

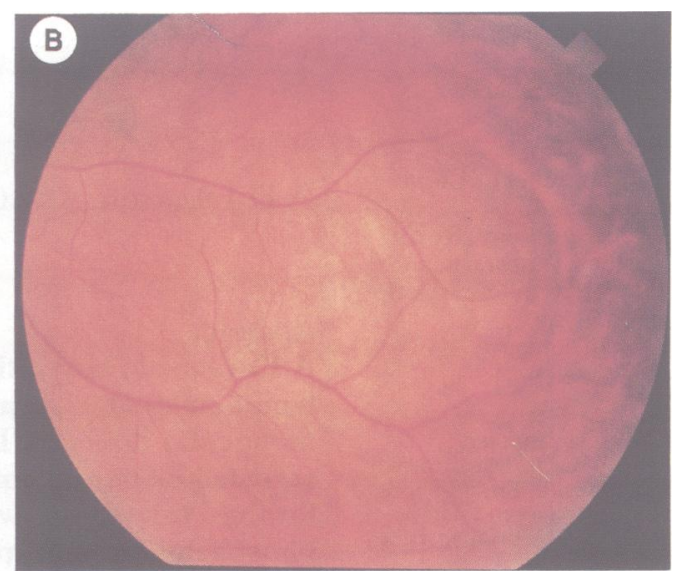

Figure $4 B$

Figure 4 Choroidal inflammation in APMPPE. A: A focal area of infiltration around a choroidal vessel as seen three weeks after presentation. B: Complete resolution 10 weeks after presentation with no damage to the pigment epithelium.

While non-specific symptoms such as headache, fever, malaise, and myalgia can be associated with a cold or viral condition, they also can occur as part of a vasculitic syndrome. ${ }^{22}$ It is possible that in some cases the headache, fever and malaise are manifestations of a transitory systemic vasculitis, of which APMPPE is a part. This vasculitis could account for the observation of cerebral vasculitis, retinal vasculitis, erythema nodosum, and in our case choroidal vasculitis in APMPPE. Although the finding of choroidal vascular infiltration in this patient does not prove that choroidal vasculitis is a requisite for APMPPE, it does lend support to the hypothesis that choroidal vasculitis plays a part in its pathogenesis.

The authors recently examined a second consecutive case of APMPPE after this report was written. While the disease was active scattered infiltrates around the choroidal vessels were found and these infiltrates appeared similar to those described in this report. The infiltrates resolved as the posterior pole lesions regressed. There was no abnormality of the overlying retinal pigment epithelium after the resolution of the infiltration.

1 Gass JDM. Acute posterior multifocal placoid pigment epitheliopathy. Arch Ophthalmol 1968; 80: 177-85.

2 Van Buskirk EM, Lessell S, Frieman E. Pigment epitheliopathy and erythema nodosum. Arch Ophthalmol 1971; 85: pathy and
$369-72$.

3 Deutman AF, Oosterhuis JA, Boen-Tan TN, Aan De Kerk AL. Acute posterior multifocal placoid pigment epitheliopathy. Pigment epitheliopathy or choriocapillaritis. $\mathrm{Br} \mathcal{F}$ Ophthalmol 1972; 56: 863-74.

4 Kirkham TH, ffytche TJ, Sanders MD. Placoid pigment epitheliopathy with retinal vasculitis and papillitis. $\mathrm{Br} \mathcal{F}$ Ophthalmol 1972; 56: 875-80.

5 Isashiki M, Koide H, Yamashita T, Ohba N. Acute posterior multifocal placoid pigment epitheliopathy associated with diffuse retinal vasculitis and late haemorrhagic macular detachment. Br f Ophthalmol 1986; 70: 255-9
6 Gass JDM. Acute posterior multifocal placoid pigment epitheliopathy: a long-term follow-up study. In: Fine SL, Owens SLK, eds. Management of retinal vascular and macular disorders. Baltimore: Williams and Wilkins, 1983: 176-81.

7 Lyness AL, Bird AC. Recurrences of acute posterior multifocal placoid pigment epitheliopathy. Am $\mathcal{F}$ Ophthalmol 1984; 98: 203-7.

8 Sigelman J, Behrens M, Hilal S. Acute posterior multifocal placoid pigment epitheliopathy associated with cerebra vasculitis and homonymous hemianopia. Am $\mathcal{F}$ Ophthalmol 1979; 88: 919-24.

9 Wilson CA, Choromokos EA, Sheppard R. Acute posterior multifocal placoid pigment epitheliopathy and cerebral multifocal placoid pigment epitheliopathy and
vasculitis. Arch Ophthalmol 1988; 106: 796-800.

10 Torczynski E. Choroid and suprachoroid. In: Duane TD, Jaeger EA eds. Biomedical foundations of ophthalmology. Philadelphia: Lippincott, 1988: 1-33.

11 Frohman LP, Klug R, Bielory L, Patti JC, Noble KG. Acute posterior multifocal placoid pigment epitheliopathy with unilateral retinal lesions and bilateral disk edema. $A m \mathcal{F}$ Ophthalmol 1987; 104: 548-50.

12 Priluck IA, Robertson DM, Buettner H. Acute posterior multifocal placoid pigment epitheliopathy. Urinary findings. Arch Ophthalmol 1981; 99: 1560-2.

13 Savino PJ, Weinberg RJ, Yassin JG, Pilkerton AR. Diverse manifestations of acute posterior multifocal placoid pigment epitheliopathy. Am F Ophthalmol 1974; 77: 659-62.

14 Holt WS, Regan CDJ, Trempe C. Acute posterior multifocal placoid pigment epitheliopathy. Am $\mathcal{F}$ Ophthalmol 1976; 81: placoid pign 12 .

15 Fishman GA, Baskin M, Jednock N. Spinal fluid pleocytosis in acute posterior multifocal placoid pigment epitheliopathy. Ann Ophthalmol 1977; 9: 33-6.

16 Ryan SJ, Maumenee AE. Acute posterior multifocal placoid pigment epitheliopathy. Am $\mathcal{F}$ Ophthalmol 1972; 74: 106674.

17 Clearkin LG, Hung SO. Acute posterior multifocal placoid pigment epitheliopathy associated with transient hearing loss. Trans Ophthalmol Soc UK 1983; 103: 562-4.

18 Hedges TR, Sinclair SH, Gragoudas ES. Evidence for vasculitis in acute posterior multifocal placoid pigment epitheliopathy. Ann Ophthalmol 1979; 11: 539-42.

19 Damato BE, Nanjiani M, Foulds WS. Acute posterior multifocal placoid pigment epitheliopathy. A follow up study. Trans Ophthalmol Soc UK 1983; 103: 517-22.

20 Azar P Jr, Gohd RS, Waltman D, Gitter KA. Acute posterior multifocal placoid pigment epitheliopathy associated with an
mather adenovirus type 5 infection. Am $\mathcal{F}$ Ophthalmol 1975; 80: 1003-5.

21 Laatikan LT, Immonen IJR. Acute posterior multifocal placoid pigment epitheliopathy in connection with acute nephritis. Retina 1988; 8: 122-4.

22 Fauci AS. The vasculitis syndromes. In: Braunwald E, Isselbacher KJ, Petersdorf RG, Wilson JD, Martin JB, Fauci AS, eds. Harrison's principles of internal medicine. 11th ed. New York: McGraw-Hill, 1987: 1438-45. 